\title{
Effectiveness of treatments for acute and sub-acute mechanical non-specific low back pain: protocol for a systematic review and network meta-analysis
}

Silvia Gianola ${ }^{1 *}$ D, Greta Castellini ${ }^{1,2}$, Anita Andreano ${ }^{3,4}$, Davide Corbetta ${ }^{5,6}$, Pamela Frigerio $^{7}$, Valentina Pecoraro ${ }^{8}$, Valentina Redaelli ${ }^{9}$, Andrea Tettamanti ${ }^{5,6}$, Andrea Turolla ${ }^{10}$, Lorenzo Moja ${ }^{1,2}$ and Maria Grazia Valsecchi ${ }^{3}$

\begin{abstract}
Background: Non-specific low back pain (LBP) is the leading cause of disability worldwide. Acute LBP usually has a good prognosis, with rapid improvement within the first 6 weeks. However, the majority of patients develop chronic LBP and suffer from recurrences. For clinical management, a plethora of treatments is currently available but evidence of the most effective options is lacking. The objective of this study will be to identify the most effective interventions to relieve pain and reduce disability in acute and sub-acute non-specific LBP.

Methods/design: We will search electronic databases (MEDLINE, Embase, CENTRAL) from inception onwards. The eligible population will be individuals with non-specific LBP older than 18 years, both males and females, who experience pain less than 6 weeks (acute) or between 6 and 12 weeks (subacute). Eligible interventions and comparators will include all conservative rehabilitation or pharmacological treatments provided by any health professional; the only eligible study design will be a randomized controlled trial. The primary outcomes will be pain intensity and back-specific functional status. Secondary outcomes will be any adverse events. Studies published in languages other than English will also potentially be included. Two reviewers will independently screen the titles and abstracts retrieved from a literature search, as well as potentially relevant full-text articles. General characteristics, potential effect modifiers, and outcome data will be extracted from the included studies, and the risk of bias will be appraised. Conflicts at all levels of screening and abstraction will be resolved through team discussions. After describing the results of the review, if appropriate, a random effects meta-analysis and network meta-analysis will be conducted in a frequentist setting, assuming equal heterogeneity across all treatment comparisons and accounting for correlations induced by multi-arm studies using a multivariate normal model.
\end{abstract}

Discussion: Our systematic review will address the uncertainties in the use of pharmacological or non-pharmacological treatments, and their relative efficacy, for acute and subacute LBP. These findings will be useful for patients, healthcare providers, and policymakers.

Systematic review registration: PROSPERO CRD42018102527

\footnotetext{
* Correspondence: silvia.gianola@grupposandonato.it

${ }^{1}$ Unit of Clinical Epidemiology, IRCCS Istituto Ortopedico Galeazzi, Milan, Italy

Full list of author information is available at the end of the article
}

(c) The Author(s). 2019 Open Access This article is distributed under the terms of the Creative Commons Attribution 4.0 International License (http://creativecommons.org/licenses/by/4.0/), which permits unrestricted use, distribution, and reproduction in any medium, provided you give appropriate credit to the original author(s) and the source, provide a link to the Creative Commons license, and indicate if changes were made. The Creative Commons Public Domain Dedication waiver (http://creativecommons.org/publicdomain/zero/1.0/) applies to the data made available in this article, unless otherwise stated. 


\section{Background}

Low back pain (LBP) is considered a symptom, and not a disease [1]. Various spinal structures including ligaments, facet joints, paravertebral musculature and fascia, intervertebral discs, and spinal nerve roots have been implicated as pain generators [2]. Nevertheless, $85 \%$ of patients with isolated back pain still do not have a definitive cause identified for their symptoms [3]. The aetiologies can be subdivided into mechanical, systemic, and referred groups. By far, the most frequent cause is mechanical (97\%) [2] with the most common form of "non-specific LBP" [4]. This definition is used when the cause of the pain cannot be precisely determined [1] and is based on the exclusion of patients with a specific cause (e.g., fracture, infection, cancer) [4].

Non-specific LBP is commonly defined as pain or discomfort localized in the area of the posterior aspect of the body, from the lower margin of the twelfth rib to the lower gluteal folds, with or without pain referred into one or both lower limbs, which lasts for at least 1 day [5]. Non-specific LBP is classified by the duration as acute (pain lasting less than 6 weeks), sub-acute (6 to 12 weeks), or chronic (more than 12 weeks) [6]. Acute LBP is one of the most common reasons for adults to see a general practitioner because of moderate to severe pain and debilitating motor and psychological functions [7]. The worldwide point prevalence of LBP is $9.4 \%$ (95\% CI, 9.0-9.8) in 2010 and is higher in males and the elderly, exceeding $30 \%$ in 80-year-old men in Europe [5].

Despite its widespread occurrence, acute LBP is considered to be typically self-limiting, with a recovery rate of $90 \%$ within 6 weeks of the initial episode [8], whereas 2 to $7 \%$ of patients develop chronic LBP and have a high risk of recurrence $[4,8]$. The progression to chronicity is associated with high disability and costs for society [9]. Out of all 291 conditions studied in the Global Burden of Disease 2010 Study, LBP ranked highest in terms of disability and sixth in terms of overall burden expressed as disability-adjusted life-year (DALYs). Estimated DALYs increased from 58.2 million in 1990 to 83.0 million in 2010 [5]. In fact, LBP leads to a greater number of people leaving the labor force than diabetes, hypertension, neoplasm, asthma, and heart and respiratory disease combined [10].

There are many different therapeutic interventions for acute and sub-acute non-specific LBP, including pharmacological and physiotherapy treatments that are sustained by several systematic reviews [11-22]. However, none of them has been universally accepted as being the most efficacious. The five most recent guidelines (from 2015 to 2018) developed inconsistent and discordant recommendations for acute LBP [23, 24]. The uncertainties regarding the most effective treatment may be due to the absence of multiple direct comparisons of the available treatments. In fact, the majority of published studies compare only two interventions at a time. It would be helpful for clinicians, patients, and all stakeholders to know the relative efficacy of all the available treatments for acute LBP in terms of benefits and harm, in order to inform treatment decision and let to choose the best option on the basis of evidence and not only according to expert opinion.

We therefore plan to carry out a comprehensive systematic review of the acute non-specific LBP interventions to evaluate, through a multiple-treatment meta-analysis, the contribution of the current therapeutic options used to treat these patients and offer a rank of being the best among the available treatments.

The objective of this systematic review will be to assess the effectiveness of treatments for acute and sub-acute mechanical in adults with LBP.

\section{Methods}

A systematic review protocol has been developed and registered with the PROSPERO database (CRD42018102527). This review protocol was prepared using the Preferred Reporting Items for Systematic Review and Meta-Analyses Protocol (PRISMA-P) guidelines and their recommendations $[25,26]$. We have completed the PRISMA-P checklist (Additional file 1). Additional sections specific to NMA have been considered according to Chaimani et al. [27]. We will use the PRISMA-NMA extension statement to structure the contents of the actual systematic review and network meta-analysis [26].

\section{Eligibility criteria \\ Types of studies}

We will only include randomized controlled trials (RCTs) excluding quasi-randomized trials. Cross-over randomized trials will be excluded since they are inappropriate study design for acute mechanical LBP. Studies will be considered as RCT if authors explicitly state that it is randomized [28].

\section{Participants}

We will include trials that involve participants older than 18 years, both males and females, experiencing pain for up to 12 weeks of non-specific LBP. We will classify the population based on pain duration: acute (less than 6 weeks) or subacute (6 to 12 weeks) [4]. Accordingly, we will select trials for pain duration, regardless of the population definition reported for a study (e.g., chronic patients with pain for less than 12 weeks). When the duration of pain allowed in the primary study, as the inclusion criteria, exceeds for a few weeks the standard definition of subacute pain (i.e., recruitment from 8 to 16 weeks) and the appropriate subgroup data is not reported in the publication as a subgroup, we will contact the authors to obtain the data for our population of 
interest only. If the investigators will not provide the data, the study will be excluded. According to the definition of aspecific LBP, we will exclude studies focusing on specific pathological entities (e.g., spondylolisthesis) and subgroups of patients (e.g., pregnant women). There will be no restriction on the severity or stage of the symptoms. Studies focusing on both neck and back pain in which the two subgroups of patients cannot be identified, or patients presenting with both conditions, will be excluded.

\section{Interventions}

We will consider all conservative rehabilitation or pharmacological treatments provided by health professionals, such as general medical practitioners or physiotherapists, aimed at relieving pain and/or reducing physical disability. We will consider any modality (e.g., physical, pharmacological), treatment extent, frequency, or intensity. We will exclude RCTs or arms of RCTs including non-conservative treatments (e.g., surgical approaches), herbal medicine, homeopathy, and all alternative treatments except for acupuncture and dry needling. We will include them since they could be clinically relevant for LBP stakeholders and there is sparse evidence of their efficacy for acute LBP in the literature [22, 29-31]. We will extract sufficient and important intervention details as suggested by the TIDieR checklist [32] in order to create consistent nodes [33-35]. Thus, we will set the following classification of interventions for potential nodes:

1. Biopsychosocial rehabilitation (including cognitive behavioral treatment and back school)

2. Exercise (e.g., resistance or aerobic training)

3. Manual therapy (e.g., spinal manipulation, mobilization, trigger point/myofascial therapy)

4. Dry needling and acupuncture

5. Education (e.g., booklet)

6. Any physical therapy (e.g., low-laser therapy, diathermy, transcutaneous electrical nerve stimulation, ultrasound therapy, heat wrap)

7. Taping (e.g., kinesiotaping)

8. Usual care defined as treatment suggested by general medicine (minimal intervention: advice to stay active or to take drugs as needed)

9. Paracetamol

10. Non-steroidal anti-inflammatory drugs (NSAIDs), including COX-2 inhibitors

11. Muscle relaxant drugs

12. Opioid drugs

13. Steroids

14. Antidepressant drugs

15. Inert treatment (e.g., placebo drug, sham therapy)

16. No treatment (no treatment, waiting list control)
For the exercise, education, manual therapy, and physical therapies nodes, we will explore any kind of therapy with own delivered modality. If enough studies share the same description of the intervention (assessment made by the TIDieR checklist [32]) that allows the creation of a new node, we will build a new subgroup category as follows: (I) exercise: as example, for instance, single supervised or home exercise, stretching, aerobics, or resistant training; (II) manual therapies: as example, for instance, mobilization and manipulation, trigger points, and muscle therapy; (III) education: as example, for instance, booklet/advices, ergonomics, and workplace interventions; (IV) physical therapies: as example, for instance, low-laser therapy, diathermy, transcutaneous electrical nerve stimulation, ultrasound therapy, and heat wrap.

\section{Outcomes and study time points}

Primary outcomes will be pain intensity (e.g., measured by numeric rating scale, visual analog scale, McGill Pain Questionnaire or, box scale, other validated quantitative measures) and back-specific functional status (e.g., measured by the Oswestry disability questionnaire, RolandMorris disability scale or other validated quantitative measures). If a trial reports more than one measure of pain intensity in different conditions (e.g., "night" or "at rest" or "at movement"), we will select "pain at rest" as a measure of generic pain. The secondary outcome will be any adverse event. We will define the adverse events (AE) based on the grade of severity. The Common Terminology Criteria for Adverse Events displays grades 1 through 5 with unique clinical descriptions of severity for each $\mathrm{AE}$ based on this general guideline: grade 1 mild $\mathrm{AE}$, grade 2 moderate $\mathrm{AE}$, grade 3 severe $\mathrm{AE}$, grade 4 life-threatening or disabling $\mathrm{AE}$, grade 5 death related to $\mathrm{AE}$. We will classify the $\mathrm{AE}$ a posterior, since we expect differences in the nature of events according to the type of intervention (pharmacological or not pharmacological) [36].

All time points will be abstracted. However, in the analyses, we plan to summarize the immediate-term (closest to 1 week), short-term (closest to 1 month assessment), intermediate (closest to 3-6 months), and longterm effects (closest to 12 months).

\section{Information sources}

We will search the following electronic databases since the inception date up to February 27, 2019: MEDLINE (PubMed), CENTRAL, and EMBASE (Elsevier, EMBASE. com) using the appropriate thesaurus and free-text terms. We will contact investigators and relevant trial authors, seeking information on unpublished data, if necessary.

We will check the reference lists of all the studies identified, and we will examine the references of any 
systematic review or meta-analysis identified during the search process.

No restriction on language or publication period will be applied. Non-English studies for which a translation cannot be obtained will be classed as potentially eligible but will not be considered in the full review. A full electronic search strategy for PubMed/MEDLINE is presented in Additional file 2.

\section{Study selection}

Two of the authors of the present protocol will independently screen the abstracts of all the publications obtained by the search strategy. These authors will then independently assess the full text of the potentially relevant studies for inclusion. We will discard all studies that do not fulfill the above inclusion criteria. We will then obtain the full text of the remaining articles. We will resolve disagreements through discussion and consult a third author if disagreements persist. Covidence software [37] will be used to manage the study selection phase.

\section{Data extraction}

We will use a specifically designed and piloted data collection form using an Excel sheet (Microsoft Inc.). Two authors will independently extract characteristics and outcome data from the included studies. Disagreements will be resolved through discussion or with assistance from a third author if necessary.

From each study included, we will extract the following variables expressed in PICO terms: Population definition (acute/subacute), number, gender and age of participants, dropouts; Interventions and Controls with details of treatment description (such as duration of whole treatment); and Outcomes (primary and secondary) with relative measurement scales and time point follow-up. Moreover, we will extract the following trial characteristics: name of the first author, year of publication, setting, number of centers, and funding sources.

All relevant arm-level data will be extracted. For pain and disability outcomes, we will consider post-treatment assessments. When these are lacking, the post-treatment data will be extrapolated by the difference between the baseline and mean change values and SDs will be imputed using the average of the available SD for the same instrument within the same network [38]. If there will be enough information, we will perform a secondary analysis using mean change and discuss possible differences. The AEs will be extracted as absolute number when available.

We will assume that any patient meeting the inclusion criteria is, in principle, equally likely to be randomized to any of the eligible low back pain interventions.

\section{Geometry and feasibility of the network}

We will explicitly describe the process leading to node grouping [39, 40]. The network of treatments will be judged based on the characteristics of the available studies, presented and evaluated graphically. We will evaluate the following: if the network is disconnected; if there is a sufficient number of comparisons in the network with available direct data; if there is a high number of comparisons based on a single study; and if any key treatment is missing. Next, the feasibility of the network meta-analysis will be assessed checking the following: (i) transitivity (i.e., comparable distribution of effect modifiers across comparisons), which will be examined using boxplots or percentages to visually inspect potential effect modifiers of treatment effect [41]; (ii) consistency between direct and indirect estimates of the effects, which will be examined using the node-splitting method [42], and globally (i.e., evaluating the network as a whole), using the design-bytreatment interaction model [43]; and (iii) the amount of variability, which we will quantify, that can be attributed to heterogeneity and inconsistency rather than sampling error, by calculating the $I^{2}$ statistic [44].

All RCTs reporting only two arm comparisons between the same kind of intervention (e.g., exercise versus exercise) will be excluded, whereas if they present at least one third arm comparator, they will be included (e.g., exercise versus NSAIDs). We will include both multi-arm trials comparing three or more interventions and those comparing different dosages or regimens of an intervention to a different one. Intervention arms of different dosages and regimens of the same intervention will be merged together for the global analysis of all outcomes. We will not consider all the comparisons in which an intervention presents multiple co-interventions for the experimental group (e.g., mixed treatment: laser therapy plus manipulation plus exercise versus waiting list controls) or for the control group (e.g., usual care: education, some physical exercise plus drugs taken as needed) to avoid inconsistencies across trials.

\section{Risk of bias within individual studies}

Two review authors will independently assess the risk of bias in the included studies. Disagreements will be resolved through discussion or arbitration with a third review author when consensus cannot be reached. We will assess the risk of bias for each included study using the "risk of bias" (RoB) assessment tool recommended by The Cochrane Collaboration [28]. Specifically, we will evaluate the following criteria: random sequence generation, allocation concealment, blinding of participants, providers and outcome assessment, incomplete outcome data, and selective outcome reporting. Each item will be scored as "high," "low," or "unclear" RoB if no sufficient information is reported. To summarize the overall RoB 
for a study, allocation concealment, blinding of outcome assessment, and incomplete outcome data will be carefully considered in order to classify each study as "low risk of bias" when all three criteria are met, "high risk of bias" when at least one criterion is unmet, and "moderate risk of bias" in the remaining cases. Allocation concealment, blinding of outcome assessment, and incomplete outcome data are not expected to vary in importance across the primary outcomes, and therefore, we will summarize the RoB of each study. RoB information will be used to interpret how risk of bias can affect data per each comparison in the network plot and in the interpretation of the quality of evidence.

\section{Quality of evidence}

We will assess the certainty of evidence contributing to network estimate of the main outcomes with the Grading of Recommendation Assessment, Development and Evaluation (GRADE) framework. We considered the five GRADE domains: study limitations for RoB assessment, indirectness, inconsistency, imprecision, and publication bias [45].

\section{Measures of treatment effect Methods for direct treatment comparisons}

We will perform conventional pairwise meta-analyses for each primary outcome using a random-effects model for each treatment comparison with at least two studies [46] using Stata software v. 15 and the command metan [47].

We will estimate the primary outcomes as continuous outcomes, using the mean difference (MD) or standardized mean difference (SMD) when different outcome measurements have been reported for each trial. The uncertainty of all estimates will be expressed with its 95\% confidence interval (CI).

\section{Methods for multiple comparisons}

We will estimate the primary outcomes as continuous outcomes, using standardized mean difference (SMD) as we expect multiple scales to be used. We will perform the network meta-analyses within a frequentist setting, assuming equal heterogeneity across all treatment comparisons and accounting for correlations induced by multi-arm studies $[41,48]$. We will use a multivariate normal model with random effects [43]. We will first fit a design by treatment interaction model to assess the presence of inconsistency (global $\chi^{2}$ test). If the null hypothesis of all inconsistency parameters being equal zero is not rejected, we will fit a consistency model. If a global significant inconsistency is found, we will try to interpret the significant inconsistency parameters, split nodes to possibly remove the problem, and try to model the inconsistency using meta-regression. If there will be enough information, we will perform a secondary analysis using mean change and discuss possible differences.

\section{Relative treatment ranking}

We will estimate all ranking probabilities and cumulative ranking probabilities for each treatment and outcome. We will then calculate the median rank with their 95\% credible intervals, to assess the robustness of the finding. To determine a treatment hierarchy with a single number, we will calculate the surface under the cumulative ranking curve (SUCRA) and express it as a percentage [49]. Presenting the results with this method will help to visualize the relative efficacy of treatments, as it will provide the probabilities for a treatment to be $i$ th ranking (i.e., first, second, third), for each possible rank, in improving the outcome of interest. We will perform network meta-analyses in Stata 15 [47] using the "network" command and the "mvmeta" command [43, 50-52].

\section{Assessment of statistical heterogeneity}

In the standard pairwise comparisons, we will assess the statistical heterogeneity within each pairwise comparison using the $I^{2}$ statistic, where an $I^{2}$ value of 25 to $49 \%$ indicates a low degree of heterogeneity, 50 to $75 \%$ a moderate degree of heterogeneity, and more than $75 \%$ indicates a high degree of heterogeneity [53].

In the network meta-analyses, we will assume that the standard heterogeneity is constant across the different treatment comparisons. We will estimate it including a random effect in the multivariate normal model, assuming a multivariate normal distribution with mean 0 and a variance-covariance matrix with diagonal elements $\tau^{2}$ and off-diagonal elements equal to $\tau^{2} / 2$ and discuss the magnitude of the estimated variance parameter.

\section{Assessment of transitivity and statistical consistency in network meta-analyses}

We will assess the assumption of transitivity (or similarity) by comparing the distribution of the potential effect modifiers across the various pairwise comparisons. If there are no multi-arm trials, we will evaluate the inconsistency assumption in each closed loop of the network separately as the difference between direct and indirect estimates for a specific comparison (inconsistency factor). The magnitude of the inconsistency factors and their 95\% CIs will be used to make an inference about the presence of inconsistency in each loop.

If multi-arm trials are present, as it is problematical to identify loop inconsistencies, we will use the node-splitting approach to evaluate existing differences between direct and indirect estimates for each node [42].

To check the assumption of consistency in the entire network, we will use the design-by-treatment model as described by Higgins [43]. This method accounts both 
for loop and design (i.e., different sets of treatments compared in a trial) inconsistencies in multi-arm trials. Using this approach, we will make an inference about the presence of inconsistency from any source in the entire network based on an $X^{2}$ test. Inconsistency and heterogeneity are interwoven: to distinguish between these two sources of variability, we will employ the $I^{2}$ statistic for inconsistency, as it measures the percentage of variability that cannot be attributed to random error or heterogeneity (within comparison variability).

If heterogeneity is identified and at least 10 studies are present, we will conduct a meta-regression analysis to explain the observed heterogeneity [54]. The meta-regression analysis will explore the following factors as the most likely sources of inconsistency between direct and indirect evidence: baseline pain values (source of statistical heterogeneity); age, gender, patients with acute and subacute pain (sources of clinical heterogeneity); and study quality (source of methodological heterogeneity).

\section{Sensitivity analysis}

We will provide sensitive analysis in the situation when (i) outlying studies are present and suspected and (ii) studies are arbitrarily grouped. Moreover, in case of more than 10 studies available, we will assess a small study effect for each outcome providing an adjusted funnel plot and using netfunnel command (Stata 15.0 software).

\section{Discussion}

Our systematic review results will have a direct impact on a large proportion of the population affected by nonspecific LBP since this is the most leading cause of disability worldwide. The comparative efficacy among different therapeutic interventions for acute non-specific LBP, including pharmacological and physiotherapy treatments, is currently unknown. Indeed, the results will influence therapeutic strategies for patients with LBP, policymakers and all stakeholders.

Our review has several strengths including (I) exploring a wider range of literature databases including eligible articles in all languages, (II) a transparent reporting of description of interventions for a consistent node decision making, and (III) the plan to present summary assessments using the GRADE approach to rate the quality of evidence ensuring transparent reporting and clearer interpretation of results.

We anticipate that our included interventions can be a proxy of the actual clinical practice since we will be highly selective excluding all combined interventions. Indeed, the inclusions of mixed interventions (i.e., ultrasound plus exercise) can be uninformative of which is the effective part of the treatment. This is a common problem of complex non-pharmacological interventions $[55,56]$. Potential issues of the proposed review include high clinical heterogeneity, poor quality of reporting of the included trials, and difficulty in interpreting measures of effect when the pooled estimates come from trials that measured the outcome using different measurement tools $[35,57]$. Another plausible limitation, solely concerning the network meta-analysis, might be the lack of available treatment comparisons to build robust nodes.

Any important protocol amendments will be transparently documented. We aim to disseminate the results of the NMA: we will publish the findings in an open access journal, present them at scientific conferences, and conduct dissemination meetings with key stakeholders (including policymakers and healthcare providers). We will also consider dissemination through social media tools.

\section{Additional files}

Additional file 1: PRISMA-P checklist. (DOCX $30 \mathrm{~kb}$ )

Additional file 2: PubMed search strategy and adapted for the other databases. (DOCX $14 \mathrm{~kb}$ )

\section{Abbreviations}

DALYs: Disability-adjusted life-year; LBP: Low back pain; MD: Mean difference; NMA: Network meta-analysis; NSAIDs: Non-steroidal anti-inflammatory drugs; PRISMA-P: Preferred Reporting Items for Systematic Reviews and MetaAnalyses Protocol; RCT: Randomized controlled trial; RoB: Risk of bias; SD: Standard deviation; SMD: Standardized mean difference; SUCRA: Surface under cumulative ranking area

\section{Authors' contributions}

SG and LM conceived the idea. SG and CG designed the study, developed the search strategies, and wrote the first draft protocol. AA, GV, and LM provided input into the design. All authors provided feedback on early and advanced drafts of the protocol and approved the final version. SG registered the protocol. All authors read and approved the final manuscript.

\section{Funding}

The work was supported by two grants from the Italian Ministry of Health (Ricerca Corrente L3026 and Giovani Ricercatori GR-2011-02348048). The funding sources had no controlling role in the study design, data collection, analysis, interpretation, or report writing.

\section{Competing interests}

The authors declare that they have no competing interests.

\section{Author details}

${ }^{1}$ Unit of Clinical Epidemiology, IRCCS Istituto Ortopedico Galeazzi, Milan, Italy. ${ }^{2}$ Department of Biomedical Sciences for Health, University of Milan, Milan, Italy. ${ }^{3}$ Center of Biostatistics for Clinical Epidemiology, School of Medicine and Surgery, University of Milano-Bicocca, Monza, Italy. ${ }^{4}$ Epidemiology Unit, Agency for Health Protection of Milan, Milan, Italy. ${ }^{5}$ Rehabilitation and Functional Recovery Department, IRCCS San Raffaele Hospital, Milan, Italy. ${ }^{6}$ Vita-Salute San Raffaele University, Milan, Italy. ${ }^{7}$ Child and Adolescent Neuropsychiatric Unit, ASST Grande Ospedale Metropolitano Niguarda, Milan, Italy. ${ }^{8}$ Department of Laboratory Medicine, OCSAE, Azienda USL of Modena, Modena, Italy. ${ }^{9}$ Casa di Cura del Policlinico di Milano, Milan, Italy.

${ }^{10}$ Laboratory for Neurorehabilitation Technologies, Fondazione Ospedale San Camillo IRCCS, Venice, Italy. 
Received: 21 August 2018 Accepted: 24 July 2019

\section{Published online: 08 August 2019}

\section{References}

1. Maher C, Underwood M, Buchbinder R. Non-specific low back pain. Lancet. 2016.

2. Deyo RA, Weinstein JN. Low back pain. The New England journal of medicine. 2001;344(5):363-70.

3. Frymoyer JW. Back pain and sciatica. The New England journal of medicine. 1988;318(5):291-300.

4. van Tulder M, Becker A, Bekkering T, Breen A, del Real MT, Hutchinson A, et al. Chapter 3. European guidelines for the management of acute nonspecific low back pain in primary care. Eur Spine J. 2006;15(Suppl 2): S169-91.

5. Hoy D, March L, Brooks P, Blyth F, Woolf A, Bain C, et al. The global burden of low back pain: estimates from the Global Burden of Disease 2010 study. Annals of the rheumatic diseases. 2014;73(6):968-74.

6. Koes BW, van Tulder M, Lin CW, Macedo LG, McAuley J, Maher C. An updated overview of clinical guidelines for the management of non-specific low back pain in primary care. Eur Spine J. 2010;19(12):2075-94.

7. Casazza BA. Diagnosis and treatment of acute low back pain. American Family Physician. 2012;85(4):343-50.

8. da C, Menezes Costa L, Maher CG, Hancock MJ, McAuley JH, Herbert RD, Costa LO. The prognosis of acute and persistent low-back pain: a metaanalysis. CMAJ. 2012;184(11):E613-24.

9. van Tulder MW, Koes BW, Bouter LM. A cost-of-illness study of back pain in The Netherlands. Pain. 1995;62(2):233-40.

10. Schofield P. Assessment and management of pain in older adults with dementia: a review of current practice and future directions. Current opinion in supportive and palliative care. 2008;2(2):128-32.

11. Rubinstein SM, Terwee CB, Assendelft WJ, de Boer MR, van Tulder MW Spinal manipulative therapy for acute low-back pain. The Cochrane database of systematic reviews. 2012;9:CD008880.

12. Dahm KT, Brurberg KG, Jamtvedt G, Hagen KB. Advice to rest in bed versus advice to stay active for acute low-back pain and sciatica. The Cochrane database of systematic reviews. 2010;6:CD007612.

13. Roelofs PD, Deyo RA, Koes BW, Scholten RJ, van Tulder MW. Non-steroidal anti-inflammatory drugs for low back pain. The Cochrane database of systematic reviews. 2008;1:CD000396.

14. Marin TJ, Van Eerd D, Irvin E, Couban R, Koes BW, Malmivaara A, et al. Multidisciplinary biopsychosocial rehabilitation for subacute low back pain. The Cochrane database of systematic reviews. 2017;6:CD002193.

15. Saragiotto BT, Machado GC, Ferreira ML, Pinheiro MB, Abdel Shaheed C, Maher CG. Paracetamol for low back pain. The Cochrane database of systematic reviews. 2016;6:CD012230.

16. Poquet N, Lin CW, Heymans MW, van Tulder MW, Esmail R, Koes BW, et al. Back schools for acute and subacute non-specific low-back pain. The Cochrane database of systematic reviews. 2016:4:CD008325.

17. Macedo LG, Saragiotto BT, Yamato TP, Costa LO, Menezes Costa LC, Ostelo RW, et al. Motor control exercise for acute non-specific low back pain. The Cochrane database of systematic reviews. 2016;2:CD012085.

18. Furlan AD, Giraldo M, Baskwill A, Irvin E, Imamura M. Massage for low-back pain. The Cochrane database of systematic reviews. 2015;(9):CD001929.

19. Yousefi-Nooraie R, Schonstein E, Heidari K, Rashidian A, Pennick V, AkbariKamrani M, et al. Low level laser therapy for nonspecific low-back pain. The Cochrane database of systematic reviews. 2008;2:CD005107.

20. Engers $A$, Jellema $P$, Wensing $M$, van der Windt DA, Grol $R$, van Tulder MW. Individual patient education for low back pain. The Cochrane database of systematic reviews. 2008:1:CD004057.

21. Urquhart DM, Hoving JL, Assendelft WW, Roland M, van Tulder MW. Antidepressants for non-specific low back pain. The Cochrane database of systematic reviews. 2008;(1):CD001703.

22. Furlan AD, van Tulder MW, Cherkin DC, Tsukayama H, Lao L, Koes BW, et al. Acupuncture and dry-needling for low back pain. The Cochrane database of systematic reviews. 2005;(1):CD001351.

23. O'Connell NE, Cook CE, Wand BM, Ward SP. Clinical guidelines for low back pain: a critical review of consensus and inconsistencies across three major guidelines. Best practice \& research Clinical rheumatology. 2016;30(6):968-80.

24. Almeida M, Saragiotto B, Richards B, Maher CG. Primary care management of non-specific low back pain: key messages from recent clinical guidelines. The Medical journal of Australia. 2018;208(6):272-5.
25. Moher D, Stewart L, Shekelle P. Implementing PRISMA-P: recommendations for prospective authors. Systematic reviews. 2016;5:15.

26. Hutton B, Salanti G, Caldwell DM, Chaimani A, Schmid CH, Cameron C, et al. The PRISMA extension statement for reporting of systematic reviews incorporating network meta-analyses of health care interventions: checklist and explanations. Annals of internal medicine. 2015;162(11):777-84.

27. Chaimani A, Caldwell DM, Li T, Higgins JP, Salanti G. Additional considerations are required when preparing a protocol for a systematic review with multiple interventions. Journal of clinical epidemiology. 2017.

28. Higgins JP, Altman DG, Gotzsche PC, Juni P, Moher D, Oxman AD, et al. The Cochrane Collaboration's tool for assessing risk of bias in randomised trials. BMJ. 2011;343:d5928.

29. Liu L, Huang QM, Liu QG, Thitham N, Li LH, Ma YT, et al. Evidence for dry needling in the management of myofascial trigger points associated with low back pain: a systematic review and meta-analysis. Archives of physical medicine and rehabilitation. 2018;99(1):144-52 e2.

30. Yeganeh M, Baradaran HR, Qorbani M, Moradi Y, Dastgiri S. The effectiveness of acupuncture, acupressure and chiropractic interventions on treatment of chronic nonspecific low back pain in Iran: a systematic review and metaanalysis. Complementary therapies in clinical practice. 2017;27:11-8.

31. Hu HT, Gao H, Ma RJ, Zhao XF, Tian HF, Li L. Is dry needling effective for low back pain?: a systematic review and PRISMA-compliant meta-analysis. Medicine. 2018;97(26):e11225.

32. Hoffmann TC, Glasziou PP, Boutron I, Milne R, Perera R, Moher D, et al. Better reporting of interventions: template for intervention description and replication (TIDieR) checklist and guide. BMJ. 2014;348:91687.

33. Hoffmann TC, Oxman AD, loannidis JP, Moher D, Lasserson TJ, Tovey DI, et al. Enhancing the usability of systematic reviews by improving the consideration and description of interventions. BMJ. 2017;358:2998.

34. Mosseri J, Trinquart L, Nizard R, Ravaud P. Meta-analysis of a complex network of non-pharmacological interventions: the example of femoral neck fracture. PloS one. 2016;11(1):e0146336.

35. Gianola S, Castellini G, Agostini M, Bolotta R, Corbetta D, Frigerio P, et al. Reporting of rehabilitation intervention for low back pain in randomized controlled trials: is the treatment fully replicable? Spine. 2016;41(5):412-8.

36. CTCAE. The common terminology criteria for adverse events. https://ctep. cancer.gov/protocolDevelopment/electronic_applications/docs/ctcaev3.pdf. Accessed Feb 2018

37. COEVIDENCE. https://www.covidence.org/home. Accessed Jan 2018.

38. Higgins J, Deeks J, Altman D. Chapter 16: Special topics in statistics. In: Higgins JPT, Green S, editors. Cochrane Handbook for Systematic Reviews of Interventions. Version 510 (updated March 2011) The Cochrane Collaboration; 2011. Available from. http://handbook-5-1.cochrane.org/.

39. James A, Yavchitz A, Ravaud P, Boutron I. Node-making process in network meta-analysis of nonpharmacological treatment are poorly reported. Journal of clinical epidemiology. 2018;97:95-102.

40. James A, Yavchitz A, Boutron I. Importance of the methods used to support the node-making process in network meta-analysis. Journal of clinical epidemiology. 2018

41. Salanti G. Indirect and mixed-treatment comparison, network, or multipletreatments meta-analysis: many names, many benefits, many concerns for the next generation evidence synthesis tool. Research synthesis methods. 2012;3(2):80-97.

42. Dias S, Welton NJ, Caldwell DM, Ades AE. Checking consistency in mixed treatment comparison meta-analysis. Statistics in medicine. 2010;29(7-8):932-44.

43. White I, Barrett J, Jackson D, Higgins J. Consistency and inconsistency in network meta-analysis: model estimation using multivariate metaregression. Research synthesis methods. 2012;Jun;3(2):111-25.

44. Jackson D, Barrett JK, Rice S, White IR, Higgins JP. A design-by-treatment interaction model for network meta-analysis with random inconsistency effects. Statistics in medicine. 2014;33(21):3639-54

45. Salanti G, Del Giovane C, Chaimani A, Caldwell DM, Higgins JP. Evaluating the quality of evidence from a network meta-analysis. PloS one. 2014;9(7):e99682.

46. DerSimonian R, Laird N. Meta-analysis in clinical trials. Controlled clinical trials. 1986;7(3):177-88

47. Stata-IC. Stata Statistical Software, v 15. College Station: StataCorp LLC; 2017.

48. Miladinovic B, Hozo I, Chaimani A, Djulbegovic B. Indirect treatment comparison. Stata Journal. 2014:14(1):76-86.

49. Salanti G, Ades AE, loannidis JP. Graphical methods and numerical summaries for presenting results from multiple-treatment meta-analysis: an overview and tutorial. Journal of clinical epidemiology. 2011;64(2):163-71. 
50. Chaimani A, Higgins JP, Mavridis D, Spyridonos P, Salanti G. Graphical tools for network meta-analysis in STATA. PloS one. 2013;8(10):e76654.

51. (MTM). M-TM-a. A framework for evaluating and ranking multiple healthcare technologies. [http://www.mtm.uoi.gr/ (accessed 8 Feb 2017).].

52. White I. Multivariate random-effects meta-regression: updates to mvmeta. The STATA Journal. 2011;11:255-70.

53. Higgins JP, Thompson SG, Deeks JJ, Altman DG. Measuring inconsistency in meta-analyses. BMJ. 2003;327(7414):557-60.

54. Agency for Healthcare Research and Quality (AHRQ): Meta-regression Approaches. 2004 R, MD: Agency for Healthcare Research and Quality.

55. Boutron I, Altman DG, Moher D, Schulz KF, Ravaud P, Group CN. CONSORT Statement for Randomized Trials of Nonpharmacologic Treatments: A 2017 Update and a CONSORT Extension for Nonpharmacologic Trial Abstracts. Annals of internal medicine. 2017;167(1):40-7.

56. Boutron I, Moher D, Altman DG, Schulz KF, Ravaud P, Group C. Methods and processes of the CONSORT Group: example of an extension for trials assessing nonpharmacologic treatments. Annals of internal medicine. 2008; 148(4):W60-6.

57. Gianola S, Frigerio P, Agostini M, Bolotta R, Castellini G, Corbetta D, et al. Completeness of outcomes description reported in low back pain rehabilitation interventions: a survey of 185 randomized trials. Physiotherapy Canada Physiotherapie Canada. 2016;68(3):267-74.

\section{Publisher's Note}

Springer Nature remains neutral with regard to jurisdictional claims in published maps and institutional affiliations.

Ready to submit your research? Choose BMC and benefit from:

- fast, convenient online submission

- thorough peer review by experienced researchers in your field

- rapid publication on acceptance

- support for research data, including large and complex data types

- gold Open Access which fosters wider collaboration and increased citations

- maximum visibility for your research: over $100 \mathrm{M}$ website views per year

At $\mathrm{BMC}$, research is always in progress.

Learn more biomedcentral.com/submissions 